Note

\title{
Supplementation of Bifidobacterium longum to a High-Fat, Low-Calcium Diet Lowers Cytolytic Activity of Fecal Water in Rats Injected with 1,2-Dimethylhydrazine Dihydrochloride
}

\author{
Taishi Oda, Yasuyuki Seto ${ }^{1}$ and Honoo Hashiba ${ }^{1}$ \\ Nutritional Science Laboratory, and ${ }^{1}$ Technology and Research Institute, \\ Snow Brand Milk Products Co. Ltd., Kawagoe, Saitama 350-1165, Japan
}

(Received July 17, 1997)

\begin{abstract}
Summary The effects of supplementing Bifidobacterium longum SBT 2928 and Lactobacillus acidophilus SBT 2062 to a high-fat, low-calcium diet on bile acid concentration, fatty acid concentration, cytolytic activity and intestinal alkaline phosphatase (ALP) activity of fecal water in rats injected with and without 1,2-dimethylhydrazine dihydrochloride (DMH) were examined. Male Wistar rats at 8 weeks of age were fed a diet containing $18 \%$ coconut oil, $2 \%$ corn oil and $0.1 \%$ calcium for $15 \mathrm{~d}$. Lyophilized cultures were supplemented to test diets at a concentration of $1 \%$. The feeding of a high-fat, low-calcium diet elevated the bile acid concentration, cytolytic activity and ALP activity of fecal water as compared to the AIN-76A diet, whereas the fatty acid concentration was not changed. None of the cultures had any effect on these parameters. Furthermore, 8 week-old rats were given a single subcutaneous injection of DMH at $40 \mathrm{mg} / \mathrm{kg}$ body weight, and fed the same diets for $15 \mathrm{~d}$. The $\mathrm{DMH}$ injection had no effect on the bile acid concentration but increased the fatty acid concentration and cytolytic activity of fecal water. In contrast, ALP activity was lower in the DMH-treated rats than in the non-treated rats. The ingestion of B. longum lowered cytolytic activity but had no effect on the bile acids, fatty acids and ALP activity of fecal water. $L$. acidophilus had no effect on these parameters.
\end{abstract}

Key Words Bifidobacteria, Lactobacillus acidophilus, cytolytic activity of fecal water, butyric acid, rats

Epidemiological studies indicate that the consumption of cultured dairy products such as fermented milk and yoghurt has an inverse correlation to the risk of colon cancer (1). Goldin and Gorbach (2) reported that a dietary supplement of Lactobacillus acidophilus reduced the incidence of 1,2-dimethylhydrazine dihydrochloride (DMH)-induced colon cancer in rats. Biasco et al (3) showed that, in a three-month human trial, the administration of $L$. acidophilus and bifidobacteria reduced proliferation activity in the upper colonic crypts. Ling (1) discussed that 
this effect of the cultures was due in part to a decrease in the level of bile acids in the aqueous phase of feces. Lidbeck et al (4) reported that soluble fecal bile acids were lowered in colon cancer patients after 6 weeks of L. acidophilus supplementation.

The incidence of colon cancer is affected by heredity and dietary factors. Lipkin et al (5) found that patients with colon cancer (familial colon cancer with polyposis) had abnormally proliferative upper crypt epithelial cells. Experimental studies (5, 6) showed that DMH treatment elevated proliferation activity in the upper colonic crypt of rodents. Furthermore, high dietary fat and low calcium intake produced an increase of bile acids and fatty acids in fecal water (7). Soluble cytolytic surfactants such as bile acids and fatty acids altered colonic epithelial cells $(8)$. The feeding of a diet high in fat and phosphate and low in calcium and vitamin D to rodents developed hyperproliferation in the lower colonic crypt (9). The colonic epithelial cell proliferation and cytolytic activity of fecal water were highly correlated (8).

The purpose of this study was to clarify the effects of supplementing bifidobacteria (Bifidobacterium longum) and $L$. acidophilus to a high-fat, low-calcium diet on the bile acid concentration, fatty acid concentration and cytolytic activity of fecal water in rats treated with and without DMH. In addition, the release of the epithelial marker intestinal alkaline phosphatase (ALP) as an indicator of intestinal epitheliallysis (10), and short-chain fatty acid (SCFA) in cecal contents were studied.

\section{Materials and methods}

Samples. B. longum SBT 2928 and L. acidophilus SBT 2062 were cultured according to Tanno et al (11). The cells were harvested by centrifugation, and washed using a saline solution. After mixing with a cryoprotectant solution containing $1 \%$ sodium glutamate and $5 \%$ sucrose, the cells were lyophilized. Lyophilized cultures contained viable cells as follows (colony-forming units/g): B. longum $3 \times 10^{11}$, and $L$. acidophilus $4 \times 10^{11}$. These materials were stored at $-20^{\circ} \mathrm{C}$ until used.

Analyses. Fecal water was collected from the homogenate of freeze-dried feces and double-distilled water $(1: 3, \mathrm{w} / \mathrm{w})$ by centrifugation after extraction at $37^{\circ} \mathrm{C}$ for $2 \mathrm{~h}$. Bile acid concentration was determined with Enzabile 2 (Daiichi Kagaku Yakuhin Co., Tokyo, Japan). Fatty acid concentration was assayed with NEFA-C Test Wako (Wako Pure Chemical Industries, Tokyo, Japan). Cytolytic activity as the lysis of erythrocytes by fecal water and intestinal ALP activity of fecal water were measured according to Lapré et al (7), except rat erythrocytes were used. Each piece of data was corrected according to the following formula: Raw data $\times 3 \times(100-$ Mean of moisture of distal colon contents per group (\%))/Mean of moisture of distal colon contents per group (\%). The contents of calcium and phosphorus in the dried feces and diets were measured by inductively coupled plasma atomic emission spectrometry after decomposition as previously reported (12). Bile acids and fatty acids in the dried feces were determined according to 
Lapré et al (7). In experiment 2, cecal contents were homogenized with 9 volumes of distilled water. SCFA were analyzed using a carboxylic acid analyzer (Model S-14, Tokyo Rika, Tokyo, Japan). In experiment 3, cecal contents were homogenized with 4 volumes of buffer, and $\beta$-glucuronidase activity was assayed according to Kulkarni and Reddy (13). The enzyme activity was expressed as milligrams of phenolphthalein liberated per hour per gram content. Another aliquot of fecal suspension was added to an equal volume of distilled water, and SCFA was measured in the same manner.

Animals and diets. Male Wistar rats at 7 weeks of age were purchased from Charles River Japan Inc. (Atsugi, Japan). Animals were housed in individual polycarbonate cages with stainless steel floor mesh, and fed a nonpurified diet (CE-2, Clea Japan Inc., Tokyo, Japan) for $7 \mathrm{~d}$. In experiment 1, 16 rats were assigned to two groups ( $n=8$ /group) such that average body weights were similar among the groups. One group was supplied a high-fat, low-calcium diet for $15 \mathrm{~d}$, and the other group was given the AIN-76A diet $(14,15)$. The high-fat, low-calcium diet contained the following (\%): casein, 20; coconut oil (Fuji Oil Co., Tokyo, Japan), 18; corn oil, 2; glucose, 50; cellulose (KC flock, Clea Japan Inc.), 5; mineral mixture (7) (Oriental Yeast Co., Tokyo, Japan), 3.5; vitamin mixture (7) (Oriental Yeast Co.), 1; DL-methionine, 0.3; and choline bitartrate, 0.2. The contents of calcium and phosphorus in the high-fat, low-calcium diet were 0.10 and $0.22 \%$, respectively. The AIN-76A diet contained the following (\%): casein, 20; corn oil, 5; glucose, 65; cellulose, 5; AIN-76 mineral mixture (Oriental Yeast Co.), 3.5; AIN-76A vitamin mixture (Oriental Yeast Co.), 1; DL-methionine, 0.3; and choline bitartrate, 0.2. The contents of calcium and phosphorus in the AIN-76A diet were 0.50 and $0.54 \%$, respectively. In experiment 2, 24 rats were assigned to three groups in the same manner. They were fed a high-fat, low-calcium diet for $15 \mathrm{~d}$. To the test diets, $1 \%$ lyophilized cultures of $B$. longum and $L$. acidophilus were supplemented. Glucose was added to adjust the diets to $100 \%$. The contents of calcium and phosphorus in the test diets were 0.10 and $0.24 \%$, respectively. In experiment 3,24 rats were assigned to three groups in the same manner, and each received a single subcutaneous injection of DMH (Nacalai Tesque Inc., Kyoto, Japan) at $40 \mathrm{mg} / \mathrm{kg}$ body weight. Before injection, DMH was dissolved in a saline solution containing $1.5 \mathrm{~mm}$ disodium ethylenediaminetetraacetic acid. The solution was adjusted to $\mathrm{pH} 6.5$ and sterilized by filtration. They were fed the same diets for $15 \mathrm{~d}$. In each experiment, feces were collected quantitatively during days 11-14 and freeze-dried. After the 15-d feeding period, the rats were sacrificed and distal colon contents were collected. The contents were freeze-dried, and moisture content was measured. In experiments 2 and 3 , cecal contents were collected and kept at $-70^{\circ} \mathrm{C}$ until used.

Data and their logarithmic transforms were made by one-factor analysis of variance (ANOVA) (16). If logarithmic transformation of the data stabilized the variance, the transformed data were used (17). The data were then analyzed by Student's $t$-test for experiment 1 and Dunnett's $t$-test for experiments 2 and 3 (16). Bile acids, fatty acids, cytolytic activity and intestinal ALP activity of fecal water, 
and cecal SCFA in experiments 2 and 3 were analyzed by two-factor ANOVA to examine the influence of DMH treatment on these parameters (16).

\section{Results and discussion}

In experiment 1, bile acid levels, cytolytic activity and intestinal ALP activity of fecal water were increased in rats fed the high-fat, low-calcium diet as compared to rats given the AIN-76A diet, consistent with the report of Lapre et al (7), whereas fatty acid levels of fecal water were not changed (data not shown). It was found that the feeding of a high-fat, low-calcium diet to rats elevated soluble fecal fatty acids as compared to a high-fat, high-calcium diet (7). The fecal excretion of calcium, phosphorus and fatty acids was lower in rats fed the high-fat, low-calcium diet than in rats given the AIN-76A diet, but bile acid excretion was the same between the groups (data not shown), consistent with a previous study (7).

Neither B. longum nor L. acidophilus had any significant influence on body weight, food intake or ingested calcium in DMH-treated rats and non-treated rats (data not shown). In non-treated rats, B. longum and L. acidophilus had no effect on the bile acids, fatty acids, cytolytic activity and intestinal ALP activity of fecal water (Table 1). Two-factor ANOVA between experiments 2 and 3 revealed that $\mathrm{DMH}$ treatment increased the cytolytic activity and fatty acid levels of fecal water significantly $(p<0.05)$, but decreased intestinal ALP activity $(p<0.05)$. DMH treatment had no significant influence on the bile acid levels of fecal water. In

Table 1. Moisture of distal colon contents, soluble fecal bile acids, soluble fecal fatty acids, cytolytic activity of fecal water, and activity of epithelial marker ALP in rats injected with and without DMH and fed a high-fat, low-calcium diet containing either B. longum or L. acidophilus. ${ }^{\text {a }}$

\begin{tabular}{|c|c|c|c|c|c|}
\hline \multirow{2}{*}{$\begin{array}{l}\text { Dietary } \\
\text { group }\end{array}$} & \multirow{2}{*}{$\begin{array}{l}\text { Moisture of } \\
\text { distal colon } \\
\text { contents }(\%)\end{array}$} & \multicolumn{4}{|c|}{ Fecal water } \\
\hline & & $\begin{array}{l}\text { Bile acids } \\
(\mathrm{mmol} / \mathrm{L})\end{array}$ & $\begin{array}{l}\text { Fatty acids } \\
(\mathrm{mmol} / \mathrm{L})\end{array}$ & $\begin{array}{c}\text { Cytolytic } \\
\text { activity }(\%)^{\mathrm{b}}\end{array}$ & $\begin{array}{l}\text { Intestinal ALP } \\
\text { activity }(\mathrm{IU} / \mathrm{mL})^{\mathrm{b}}\end{array}$ \\
\hline \multicolumn{6}{|c|}{ No DMH (Experiment 2) } \\
\hline Control & $53.1 \pm 2.4$ & $2.30 \pm 0.30$ & $0.12 \pm 0.03$ & $61 \pm 17$ & $456 \pm 67$ \\
\hline B. longum & $55.8 \pm 2.3$ & $2.13 \pm 0.25$ & $0.19 \pm 0.03$ & $74 \pm 18$ & $322 \pm 59$ \\
\hline L. acidophilus & $52.5 \pm 1.4$ & $2.11 \pm 0.19$ & $0.18 \pm 0.02$ & $65 \pm 13$ & $374 \pm 60$ \\
\hline \multicolumn{6}{|c|}{ DMH (Experiment 3) } \\
\hline Control & $59.0 \pm 1.0$ & $2.64 \pm 0.38$ & $0.32 \pm 0.02$ & $151 \pm 24$ & $216 \pm 20$ \\
\hline B. longum & $58.0 \pm 1.8$ & $2.26 \pm 0.36$ & $0.33 \pm 0.03$ & $87 \pm 19^{c}$ & $216 \pm 28$ \\
\hline L. acidophilus & $55.3 \pm 2.7$ & $2.98 \pm 0.26$ & $0.33 \pm 0.05$ & $137 \pm 20$ & $183 \pm 18$ \\
\hline
\end{tabular}

${ }^{\mathrm{a}}$ Values are means $\pm \mathrm{SE}$ for 8 rats except moisture of distal colon contents in the control groups (7 rats). DMH, 1,2-dimethylhydrazine dihydrochloride. ALP, alkaline phosphatase.

${ }^{\mathrm{b}}$ Analyzed statistically after logarithmic transformation.

${ }^{\mathrm{c}}$ Significantly different from control group in each experiment $(p<0.05)$. 
treated rats, $B$. longum lowered cytolytic activity but had no effect on the bile acids, fatty acids and intestinal ALP activity of fecal water. L. acidophilus had no effect on these parameters. Van der Meer and co-workers $(18,19)$ reported that the decreased hydrophobicity of bile acids and fatty acids inhibited their cytolytic activity. Salvioli et al (20) showed that the administration of Streptococcus faecium (the lactic acid-producing microorganism) resulted in the slower formation of secondary bile acids from primary bile acids. The hydrophobicity of secondary bile acids tended to be higher than that of primary bile acids (10). In addition, fatty acids with a carbon number $>10$ were cytolytic (18). Thus, B. longum feeding on DMH-treated rats might alter the compositions of bile acids and fatty acids in the fecal water, which would result in lower cytolytic activity of fecal water. Further studies are needed to clarify the effect of $B$. longum on compositions of bile acids and fatty acids in fecal water, intestinal epitheliallysis, and colonic epithelial cell proliferation in rats treated with $\mathrm{DMH}$.

Neither B. longum nor L. acidophilus showed changes in cecal $\beta$-glucuronidase activity in DMH-treated rats (data not shown). Goldin and Gorbach (21) showed that $\beta$-glucuronidase activity in feces was lowered by the administration of $L$. acidophilus to DMH-treated rats. Kulkarni and Reddy (13) reported that $\beta$-glucuronidase activity in cecal contents was decreased by the ingestion of $B$. longum in azoxymethane-injected rats. These controversies may be due to the strain of bacteria used or the length of the study.

In non-treated rats, the ingestion of B. longum and L. acidophilus increased the fecal excretion of phosphorus significantly $(p<0.05)$, but had no change in calcium, bile acids or fatty acids (data not shown). In DMH-treated rats, B. longum had no influence on the fecal excretion of calcium, phosphorus, bile acids or fatty acids (data not shown). L. acidophilus increased phosphorus and fatty acids significantly $(p<0.05)$, but had no change in calcium or bile acids (data not shown). Lapré et al (7) reported that supplementing calcium phosphate to a high-fat, low-calcium diet increased the fecal excretion of calcium, inorganic phosphate and fatty acids but had no change in the level of bile acids excreted. They discussed that this phenomenon was due in part to the presence of fatty acid-calciumphosphate complexes in feces $(7,22,23)$. In this study, it is believed that the feeding of B. longum and L. acidophilus did not alter fatty acid-calcium-phosphate complexes in the feces because the increase of the fecal excretion of fatty acids, calcium and phosphorus was not linked after the ingestion of B. longum or L. acidophilus.

Two-factor ANOVA of the cecal SCFA between experiments 2 and 3 revealed that DMH treatment decreased lactic and acetic acids significantly $(p<0.05)$ but increased formic acid $(p<0.05)$. It is well known that intestinal bacteria produce various SCFA (24). Thus, DMH treatment might alter cecal microflora. In nontreated rats, the ingestion of $B$. longum increased lactic acid but decreased succinic and total acids (Table 2). L. acidophilus had no effect on SCFA in non-treated rats. Succinic acid is mainly produced by Bacteroides (24). Therefore, the ingestion of $B$. longum might diminish the Bacteroides count of cecal fluids in 
Table 2. Short-chain fatty acid concentrations in cecal contents of rats injected with and without DMH, and fed a high-fat, low-calcium diet containing either B. longum or L. acidophilus. ${ }^{\mathrm{a}}$

\begin{tabular}{|c|c|c|c|}
\hline \multirow{2}{*}{$\begin{array}{l}\text { Short-chain } \\
\text { fatty acids } \\
\text { (mg/g content) }\end{array}$} & \multicolumn{3}{|c|}{ Dietary group } \\
\hline & Control & B. longum & L. acidophilus \\
\hline \multicolumn{4}{|c|}{ No DMH (Experiment 2) } \\
\hline Total & $5.38 \pm 0.30$ & $4.04 \pm 0.27^{\mathrm{c}}$ & $4.76 \pm 0.31$ \\
\hline Lactic $^{\mathrm{b}}$ & $0.13 \pm 0.01$ & $0.22 \pm 0.01^{\mathrm{c}}$ & $0.17 \pm 0.01$ \\
\hline Acetic & $2.29 \pm 0.06$ & $2.05 \pm 0.14$ & $2.19 \pm 0.07$ \\
\hline Propionic & $0.46 \pm 0.02$ & $0.44 \pm 0.03$ & $0.48 \pm 0.03$ \\
\hline Succinic & $2.15 \pm 0.30$ & $0.85 \pm 0.13^{\mathrm{c}}$ & $1.50 \pm 0.29$ \\
\hline Butyric & $0.35 \pm 0.03$ & $0.49 \pm 0.06$ & $0.43 \pm 0.04$ \\
\hline Formic & $\operatorname{tr}$ & $\operatorname{tr}$ & $\overline{\operatorname{tr}}$ \\
\hline \multicolumn{4}{|c|}{ DMH (Experiment 3) } \\
\hline Total & $5.02 \pm 0.41$ & $4.68 \pm 0.34$ & $4.93 \pm 0.65$ \\
\hline Lactic & $\operatorname{tr}$ & $\overline{\operatorname{tr}}$ & $\overline{\operatorname{tr}}$ \\
\hline Acetic & $1.80 \pm 0.09$ & $2.03 \pm 0.15$ & $1.89 \pm 0.13$ \\
\hline Propionic ${ }^{\mathrm{b}}$ & $0.41 \pm 0.05$ & $0.52 \pm 0.04$ & $0.53 \pm 0.04$ \\
\hline Succinic $^{b}$ & $2.49 \pm 0.47$ & $1.59 \pm 0.39$ & $1.90 \pm 0.63$ \\
\hline Butyric $^{b}$ & $0.30 \pm 0.05$ & $0.48 \pm 0.07^{\mathrm{c}}$ & $0.53 \pm 0.05^{\mathrm{c}}$ \\
\hline Formic $^{b}$ & $0.03 \pm 0.01$ & $0.06 \pm 0.02$ & $0.09 \pm 0.04$ \\
\hline
\end{tabular}

${ }^{\mathrm{a}}$ Values are means $\pm \mathrm{SE}$ for 8 rats. Malic acid was not detected $(<0.03 \mathrm{mg} / \mathrm{g})$; $\mathrm{tr}$, trace $(<0.03 \mathrm{mg} / \mathrm{g}) ; \mathrm{DMH}, 1,2$-dimethylhydrazine dihydrochloride.

${ }^{\mathrm{b}}$ Analyzed statistically after logarithmic transformation.

${ }^{\mathrm{c}}$ Significantly different from control group in each experiment $(p<0.05)$.

non-treated rats. In treated rats, both cultures elevated butyric acid but had no influence on other SCFA. Butyric acid appears to play a critical role in maintaining colonic epithelial integrity and health, and this may be particularly important in preventing colon cancer and ulcerative colitis (25). In in vivo studies, however, data on the protective effect of butyric acid against colon cancer and ulcerative colitis are lacking (26). Freeman (27) showed a promotive effect of sodium butyrate on colon cancer in rats treated with DMH. Deschner et al (28) provided butyrate (tributyrin) to mice using azoxymethane, but it had no effect on tumor incidence. Further studies are needed to clarify the effect of butyric acid produced by intestinal fermentation on colon cancer and ulcerative colitis.

\section{REFERENCES}

1) Ling WH. 1995. Diet and colonic microflora interaction in colorectal cancer. Nutr Res 15: 439-454.

2) Goldin BR, Gorbach SL. 1980. Effect of Lactobacillus acidophilus dietary supple- 
ments on 1,2-dimethylhydrazine dihydrochloride-induced intestinal cancer in rats. $J$ Natl Cancer Inst 64: 263-265.

3) Biasco G, Paganelli GM, Brandi G, Brillanti S, Lami F, Callegari C, Gizzi G. 1991. Effect of Lactobacillus acidophilus and Bifidobacterium bifidum on rectal cell kinetics and fecal $\mathrm{pH}$. Ital $J$ Gastroenterol 23: 142.

4) Lidbeck A, Allinger UG, Orrhage KM, Ottova L, Brismar B, Gustafsson JÅ, Rafter JJ, Nord CE. 1991. Impact of Lactobacillus acidophilus supplements on the faecal microflora and soluble faecal bile acids in colon cancer patients. Microb Ecol Health Dis 4: 81-88.

5) Lipkin M, Blattner WE, Fraumeni JF Jr, Lynch HT, Deschner E, Winawer S. 1983. Tritiated thymidine $\left(\phi_{\mathrm{p}}, \phi_{\mathrm{h}}\right)$ labeling distribution as a marker for hereditary predisposition to colon cancer. Cancer Res 43: 1899-1904.

6) Thurnherr N, Deschner EE, Stonehill EH, Lipkin M. 1973. Induction of adenocarcinomas of the colon in mice by weekly injections of 1,2-dimethylhydrazine. Cancer Res 33: 940-945.

7) Lapré JA, De Vries HT, Van der Meer R. 1993. Cytotoxicity of fecal water is dependent on the type of dietary fat and is reduced by supplemental calcium phosphate in rats. $J$ Nutr 123: 578-585.

8) Lapré JA, Van der Meer R. 1992. Diet-induced increase of colonic bile acids stimulates lytic activity of fecal water and proliferation of colonic cells. Carcinogenesis 13: 41-44.

9) Newmark HL, Lipkin M, Maheshwari N. 1990. Colonic hyperplasia and hyperproliferation induced by a nutritional stress diet with four components of western-style diet. $J$ Natl Cancer Inst 82: 491-496.

10) Lapré JA, De Vries HT, Termont DSML, Kleibeuker JH, De Vries EGE, Van der Meer R. 1993. Mechanism of the protective effect of supplemental dietary calcium on cytolytic activity of fecal water. Cancer Res 53: 248-253.

11) Tanno K, Yamamoto T, Kumazawa E, Nakamura T, Takafuji S, Nakazato H. 1988. High-concentration culture of Bifidobacterium longum. Snow Brand $R \& D$ Rep 86: 141-150.

12) Analytical Methods Committee. 1960. Methods for the destruction of organic matter. Analyst 85: 643-656.

13) Kulkarni N, Reddy BS. 1994. Inhibitory effect of Bifidobacterium longum cultures on the azoxymethane-induced aberrant crypt foci formation and fecal bacterial $\beta$-glucuronidase. Proc Soc Exp Biol Med 207: 278-283.

14) American Institute of Nutrition. 1977. Report of the AIN ad hoc committee on standards for nutritional studies. $J$ Nutr 107: 1340-1348.

15) American Institute of Nutrition. 1980. Second report of the ad hoc committee on standards for nutritional studies. J Nutr 110: 1726.

16) Joyner SP, ed. 1985. SAS/STAT ${ }^{\mathrm{TM}}$ Guide for Personal Computers, 6th Ed. SAS Institute, Cary, NC, USA.

17) Sokal RR, Rohlf FJ. 1981. Descriptive statistics: The logarithmic transformation. In: Biometry, p 419-421. W. H. Freeman and Co., SF, USA.

18) Lapré JA, Termont DSML, Groen AK, Van der Meer R. 1992. Lytic effects of mixed micelles of fatty acids and bile acids. Am J Physiol 263: 333-337.

19) Van der Meer R, Termont DSML, De Vries HT. 1991. Differential effects of calcium ions and calcium phosphate on cytotoxicity of bile acids. Am J Physiol 260: 142-147.

20) Salvioli G, Salati R, Bondi M, Fratalocchi A, Sala BM, Gibertini A. 1982. Bile acid transformation by the intestinal flora and cholesterol saturation in bile: Effects of 
Streptococcus faecium administration. Digestion 23: 80-88.

21) Goldin B, Gorbach SL. 1977. Alterations in fecal microflora enzymes related to diet, age, Lactobacillus supplements, and dimethylhydrazine. Cancer 40: 2421-2426.

22) Rafter JJ, Child P, Anderson AM, Alder R, Eng V, Bruce WR. 1987. Cellular toxicity of fecal water depends on diet. Am J Clin Nutr 45: 559-563.

23) Suzuki K, Suzuki K, Mitsuoka T. 1992. Effect of low-fat, high-fat, and fibersupplemented high-fat diets on colon cancer risk factors in feces of healthy subjects. Nutr Cancer 18: 63-71.

24) Morishita Y. 1990. Function of intestinal microflora and health. In: Structure and Function of Intestinal Microflora (in Japanese), p 109-115. Asakura Shoten, Tokyo.

25) Cummings JH, Rombeau JL, Sakata T, eds. 1995. Physiological and Clinical Aspects of Short-Chain Fatty Acids. Cambridge University Press, Cambridge, UK.

26) Lupton JR. 1995. Short-chain fatty acids and colon tumorigenesis: Animal models. In: Physiological and Clinical Aspects of Short-Chain Fatty Acids (Cummings JH, Rombeau JL, Sakata T, eds), p 307-318. Cambridge University Press, Cambridge, UK.

27) Freeman HJ. 1986. Effects of differing concentrations of sodium butyrate on 1,2dimethylhydrazine-induced rat intestinal neoplasia. Gastroenterology 91: 596-602.

28) Deschner EE, Ruperto JF, Lupton JR, Newmark HL. 1990. Dietary butyrate (tributyrin) does not enhance AOM-induced colon tumorigenesis. Cancer Lett 52: 79-82. 